\title{
Chemokines as modulators of neuroendocrine functions
}

\author{
William Rostene and Julia C Buckingham ${ }^{1}$ \\ INSERM U732, Universitó Pet M Curie, Hôpital St-Antoine, 184 rue du Faubourg St-Antoine, 75571 Paris Cedex 12, France \\ ${ }^{1}$ Division of Neuroscience and Mental Health, Imperial College London, Hammersmith campus, Du Cane Road, London W12 0NN, UK \\ (Requests for offprints should be addressed to W Rostene; Email: rostene@st-antoine.inserm.fr)
}

\begin{abstract}
Chemokines are small secreted proteins with chemoattractant properties for immune cells. Besides their role in the immune system, chemokines and their receptors may play important roles in the central nervous system. Neurodegenerative disorders that involve neuroinflammation such as multiple sclerosis, stroke, Alzheimer's disease, Parkinson's disease and HIV-associated dementia are commonly associated with local upregulation and release of chemokines. However, recent work has established that certain chemokines, constitutively expressed in the brain, exert functions in the brain that are distinct from inflammation. These chemokines regulate neuronal migration during brain development, modulate neuronal activity and play a role in various neurodegenerative diseases, pain and more recently in neuroendocrine functions. All these novel aspects, mainly focused on the chemokine stromal cell-derived factor1/CXCL12 and its receptor CXCR4, were presented by pioneers in the field during the symposium held at the sixth International Congress of Neuroendocrinology in Pittsburgh, Pennsylvania, USA in June 2006.
\end{abstract}

Journal of Molecular Endocrinology (2007) 38, 351-353

The chemokines or 'chemotactic cytokines' are an important family of signalling molecules. They were first discovered as substances which assist the recruitment of leukocytes to sites of injury or infection and, thereby, modulate immune and inflammatory responses (reviewed in Pease \& Williams 2006). However, it is now apparent that the chemokines fulfil a considerably wider brief and that their chemoattractant properties are critical for the organization of cells and tissues throughout the lifespan of an organism. Chemokines thus contribute to processes such as haematopoiesis, angiogenesis, growth and tissue repair. The first chemokines to be discovered were IP-10, IL-8, regulated on activation, normal $\mathrm{T}$ expressed and secreted (RANTES), monocyte chemoattractant protein (MCP)-1 and the macrophage inhibitory proteins, macrophage inflammatory protein (MIP)-1 and MIP-2. In human beings, some forty family members of protein have now been identified. All are small proteins comprising 60-100 amino acid residues. Chemically, they fall into two groups, CC and CXC, dependent upon the juxtaposition of their two $\mathrm{N}$-terminal cysteine residues. Chemokines signal through a family of membrane-bound G-proteincoupled receptors. These receptors are subdivided into two major classes, termed CCR and CXCR, according to their selectivity for CC and CXC cytokines respectively. However, within this framework there is a degree of receptor promiscuity, as each of the 18 receptors identified so far appears to interact with several different chemokines (for further details see Pease \& Williams 2006).

Much of the work on chemokines has focused on peripheral inflammatory responses. However, evidence that chemokines and their receptors are expressed in activated astrocytes and microglia at sites of inflammatory lesions in the brain has raised the possibility that chemokines contribute to the neuroinflammatory responses observed, for example, in conditions of brain injury, stroke and Alzheimer's disease (Tran \& Miller 2003). Interestingly, a rapidly growing body of evidence suggests that chemokines and their receptors also play an important role in the healthy brain, in particular by facilitating the migration of cells during development and by acting as neuromodulators of neuroendocrine functions (Kitabgi et al. 2006). These emerging concepts were illustrated by pioneers in the field at a symposium entitled 'Chemokines as modulators of neuroendocrine functions' which was held at the sixth International Congress of Neuroendocrinology in Pittsburgh in June 2006.

The symposium focused exclusively on one chemokine, stromal cell-derived factor-1 (SDF-1), and its cognate receptor, CXCR4. SDF-1, now termed

DOI: 10.1677/JME-07-0006 Online version via http://www.endocrinology-journals.org 
CXCL12, was originally cloned from a murine bone marrow stromal cell line and identified as a factor 'which partially replaces the need of stromal cells for the generation of B cells in vivo' (Kitabgi et al. 2006). It was later also shown to be a potent chemoattractant for T-lymphocytes. There are two major species of SDF-1 (SDF-1 $\alpha$ and SDF-1 $\beta$ ), formed by alternative mRNA splicing. SDF- $1 \alpha$, a 68 -residue protein with an additional 21-residue signal peptide at its N-terminus, appears to fulfil the majority of the known biological effects of SDF-1. SDF- $1 \beta$ is identical to SDF- $1 \alpha$ but has a 4-residue extension at the C-terminus. Recently, a third mRNA variant, termed SDF- $1 \gamma$, has been described in rat brain, but the corresponding protein has yet to be characterized.

Recent immunohistochemical studies have shown that SDF-1 and CXCR4 are constitutively expressed in adult rat brain neurons in a highly localized fashion, with particularly dense staining in the hypothalamus (Kitabgi et al. 2006). SDF-1 and CXCR4 co-localize with vasopressin (AVP) in the magnocellular neurons of the supraoptic (SON) and paraventricular (PVN) hypothalamic nuclei and with melanin-concentrating hormone (MCH)-expressing neurons in the lateral hypothalamus. In the opening paper of the symposium, Stephane Melik-Parsadaniantz (INSERM, Paris, France) focused on the magnocellular system and provided novel evidence that SDF-1 modulates the activity of vasopressinergic neurons. He thus describes the co-localisation of SDF-1 $\alpha$ and CCXR4 with AVP, but not oxytocin, in magnocellular neurons projecting from the SON and PVN through the median eminence to the neurohypophysis. He also shows, using electrophysiological techniques in vivo and in vitro, that SDF- $1 \alpha$ acts in the SON via CXCR4 to modulate the firing pattern of AVP neurons. SDF- $1 \alpha$ thus suppresses the release of AVP induced either by angiotensin II or via the somatodendritic autoregulatory loop. Changes in plasma osmolarity and water balance, induced for example by salt loading, modify immunostaining of SDF1 and CXCR4 in the magnocellular vasopressinergic neurons, producing changes which parallel the pattern of AVP release. Taken together, these data suggest that SDF-1 and its receptor CXCR4 play an important role in the local (autocrine) regulation of AVP release in normal physiological conditions.

In the subsequent presentation, Alice Guyon (CNRS Sophia-Antipolis, France) discussed the influence of SDF-1 $\alpha$ on the MCH neurons and described pertinent electrophysiological data which led her to hypothesize that SDF- $1 \alpha$ is an intra-hypothalamic regulator of energy homeostasis. Her results demonstrate that SDF- $1 \alpha$ acts via CXCR4 to modulate the electrical activity of MCH neurons in slices of rat's lateral hypothalamic tissue. Low concentrations of SDF- $1 \alpha$ thus decrease the peak and discharge of action potentials in these cells and this effect is blocked by the non-peptide CXCR4 antagonist, AMD3100. As these neurons contribute to the processes regulating feeding behaviour by releasing the orexigenic peptide MCH and show decreased CXCR4 expression when food intake is increased, she argues that SDF- $1 \alpha$ may suppress food intake, and possibly also be involved in the aetiology of anorexia, by decreasing $\mathrm{MCH}$ neuronal excitability and possibly MCH release. Further studies are now needed to verify this intriguing hypothesis.

Ralf Stumm (University of Magdeburg, Germany) addressed the role of SDF- $1 \alpha$ in the regulation of brain development, presenting elegant evidence which implicates SDF1 and CXCR4 in the development of neuronal circuits in the neocortex. Using embryos from SDF1- and CXCR4-null mice, he identified a role for SDF-1 in axonal guidance and in the selective migration and localization of gamma amino butyric acid (GABA) neurons. SDF-1 and CXCR4 are both expressed as early as embryonic day 14 and the SDF- $1 \alpha$ isoform of SDF-1 predominates after embryonic day (day 17). In addition, both the chemokine and its receptor are found in adult dentate gyrus of the hippocampal formation, raising the possibility that SDF- $1 \alpha$ and CXCR4 may also have a role in neuronal cell regeneration.

In the final paper, Tullio Florio (University of Genova, Italy) provided novel evidence for the role of SDF- $1 \alpha /$ CXCR 4 in the regulation of anterior pituitary cell proliferation and hormone secretion. CXCR4 is expressed in the adenohypophysis and may be a target for SDF- $1 \alpha$ of hypothalamic origin. Using the GH4C1 cell line, which expresses CXCR4 but not SDF-1, as a model, he shows that SDF- $1 \alpha$ has mitogenic properties, acting via CXCR4 to trigger signalling pathways involving $\mathrm{Ca}^{2+}$ mobilization, mitogen activated protein kinase (MAPK) and extracellular signal regulated kinase (Erk) $1 / 2$, Pyk2 and $\mathrm{Ca}^{2+}$-activated $\mathrm{K}^{+}$-channels. SDF- $1 \alpha$ also induces growth hormone $(\mathrm{GH})$ release from these cells, apparently via a mechanism which is dependent only on $\mathrm{Ca}^{2+}$ mobilization, but not on the other signalling molecules implicated in the mitogenic action of the chemokine. Finally, he provided evidence for a possible role for SDF-1 $\alpha /$ CXCR4 in the pathogenesis of pituitary adenomas.

This is the first symposium on chemokines to be held at a major neuroendocrinology meeting. The presentations give us insight in to an exciting new field. Chemokines, it appears, have a significant role in brain development and in the repair processes which follow neurotoxic insult and/or neuroinflammation. They are also strongly implicated in the control of neuroendocrine function at the levels of the hypothalamus and anterior pituitary gland. Studies to date have focused mainly on SDF-1 $\alpha /$ CXCR4; much 
further work is now needed to crystallize our understanding of the precise roles of this chemokine and its receptor in the brain and neuroendocrine system, and to tackle the largely unchartered field of the potential role of other members of the chemokine family.

\section{Acknowledgements}

The authors declare that there is no conflict of interest that would prejudice the impartiality of this scientific work.

\section{References}

Kitabgi P, Melik-Parsadaniantz S \& Rostène W 2006 Chemokines: a new peptide family of neuromodulators. In The Handbook of Biologically Active Peptides, pp 579-585. Ed AJ Kastin. Academic Press.

Pease JE \& Williams TJ 2006 The attraction of chemokines as a target for specific anti-inflammatory therapy. British Journal of Pharmacology 147 S212-S221.

Tran PB \& Miller RJ 2003 Chemokine receptors:signposts to brain development and disease. Nature Review Neuroscience 4 444-455.

Received in final form 22 January 2007

Accepted 23 January 2007

Made available online as an Accepted Preprint 23 January 2007 